Causality and Attributions of Intentionality Predict Belief in the Teleology of Nature

\author{
Andrew J. Roberts ${ }^{1}$, Simon Handley ${ }^{2}$, and Vince Polito ${ }^{1}$ \\ ${ }^{1}$ School of Psychological Sciences, Macquarie University \\ ${ }^{2}$ Office of Higher Degree Research Training and Partnership, Macquarie University \\ DRAFT VERSION $-2^{\text {th }}$ OCTOBER 2021. PLEASE DO NOT COPY OR CITE \\ WITHOUT THE AUTHOR'S PERMISSION
}

\begin{abstract}
Author Note
This research did not receive any specific grant from funding agencies in the public, commercial, or not-for-profit sectors. We have no conflicts of interest to disclose. Supplementary materials, data, and analysis code are stored on OSF (links provided in each study). Correspondence concerning this article should be addressed to Andrew J. Roberts, School of Psychological Sciences, Macquarie University, Sydney, NSW, 2109. Email: andrew.roberts@mq.edu.au
\end{abstract}




\begin{abstract}
To explain the teleology of something is to describe its purpose. Certain things, such as goal directed behaviour or intentional design, clearly have a purpose. However, other things, such as biological and nonbiological natural entities, do not. A prominent theory of teleological reasoning suggests that teleological acceptance is driven by an early-developing understanding that intentional agents have purposes. Recently, it has been proposed that the "goodness of fit" between a structure and a function serves as a cue that teleological explanation is appropriate. Across three large pre-registered studies, we investigated how judgements of structure-function fit and individual differences in anthropomorphism and inhibition of intuitions relate to teleological acceptance (Study One), how the strength of a causal relationship relates to judgements of structure-function fit (Study Two), and how individual differences in anthropomorphism, belief in God, and inhibition of intuitions may facilitate or constrain the transition from a causal belief to teleological acceptance (Study Three). Our results suggest that questions about structure-function fit are interpreted as asking whether there is a causal relationship between a structure and a function, and that this is a necessary but not sufficient condition for teleological belief. Furthermore, our results demonstrate that belief in God and the tendency to anthropomorphise facilitate the transition from a causal relationship to a teleological belief, whereas the tendency to inhibit intuitions constrains this transition. We discuss these findings in relation to existing theoretical and empirical literature, and argue that they reveal several distinct pathways by which such teleological beliefs may be modified.
\end{abstract}




\section{Causality and Attributions of Intentionality Predict Belief in the Teleology of Nature}

Everything happens for a reason. Plants grow because they receive sunlight, rivers get to the ocean because they flow downstream, and people earn money because they go to work. However, some things also happen for a very different type of reason. Doctors prescribe antibiotics to treat infections, traffic lights change colour to control the flow of traffic, and animals have ears so they can hear. The first category of reason, in which things are explained with reference to a preceding event, could be described as mechanistic (e.g., Dennett, 2017). The second category of reason, in which things are explained with reference to a proceeding event, are teleological (e.g., Hempel \& Oppenheim, 1948; Kelemen, 1999a; Lombrozo \& Carey, 2006). From a philosophical perspective (e.g., Dennett, 2017; Wright, 1976), certain things, such as goal-directed behaviour or human-made artefacts, can legitimately be described with teleological explanations, whereas other things, such as biological and nonbiological natural entities, cannot. However, psychological research shows that people often deviate in predictable ways from normative accounts of teleological reasoning (Kelemen et al., 2013; Liquin \& Lombrozo, 2018; Lombrozo et al., 2007; Mills \& Frowley, 2014; Roberts et al., 2021; but see Greif et al., 2006; Keil, 1995). Rather than restricting teleology to things which clearly exist (or occur) to fulfil a certain function, adults (Kelemen \& Rosset, 2009; Lombrozo et al., 2007; Zemla et al., 2012), and especially children (Kelemen, 1999d, 1999c), sometimes endorse teleological explanations for things which warrant only mechanistic explanations.

\section{Domain Differences}

The appropriateness of teleology is determined, in part, by the domain of what is being explained. Perhaps the most natural and ubiquitous use of teleology is in describing the goaldirected behaviour of an intentional agent. According to Dennett (1987), people can adopt one of a number of "stances" to make sense of the world. The stance involved in making sense of the behaviour of agents, is known as the intentional stance. This stance involves "treating the 
object whose behaviour is to be predicted as a rational agent, figuring out what beliefs and desires that agent ought to have, and predicting this rational agent will act to further its goals in light of its beliefs" (Dennett, 1987, p. 17). Evidence from the field of developmental psychology suggests that a tendency to adopt an intentional stance arises early in life (Csibra, 2008; Gergely et al., 1995; Johnson et al., 2001; Meltzoff, 1995), and by predicting actions based on an agent's beliefs and desires, taking an intentional stance necessarily leads to a teleological explanation of behaviour. Teleological explanations of goal-directed behaviour are not only ubiquitous, but according to Wright (1976), appropriate. Wright (1976) argues that " $S$ does $B$ for the sake of $G$ ", if, and only if:

1. B tends to bring about $G$, and

\section{B occurs because it tends to bring about $G$}

Put differently, a subject (S) performs a behaviour (B) to reach a goal (G), if, and only if, the behaviour tends to bring about the goal, and the behaviour occurs because it tends to bring about the goal. Consider the teleological statement, "doctors prescribe antibiotics to treat infections". In this example, both criteria are met. Prescribing antibiotics (B) tends to bring about a reduction in infection $(\mathrm{G})$, and this reduction in infection $(\mathrm{G})$ is the reason why the prescription of antibiotics (B) occurs.

Another common use of teleology is to describe the functions of human-made artefacts. Rather than predicting that a rational agent will act to further its goals in light of its beliefs, Dennett (1987) argues that artefacts are understood by adopting a design stance, which involves asking what an object was designed to do. A sensitivity to the functions of artefacts arises early in development (Casler \& Kelemen, 2005, 2007; Hernik \& Csibra, 2015), and just as an intentional stance results in a teleological explanation of behaviour, a design stance necessarily results in a teleological explanation of an artefact. Like teleological explanations of behaviour, Wright (1976) argues that teleological explanations of human-made artefacts are appropriate. 
However, rather than referring to subjects, behaviours, and goals, Wright's (1976) formulation is changed slightly so that "the function of $X$ is $Z$ ", if, and only if:

1. Z is a consequence of $X$ 's being there, and

\section{2. $X$ is there because it results in $Z$}

Consider the teleological statement, "traffic lights change colour to control the flow of traffic". This example meets both criteria, as controlling the flow of traffic $(\mathrm{Z})$ is a consequence of traffic lights changing colour (X), and traffic lights are there (i.e., were designed) to change colour (X) because this results in the flow of traffic being controlled (Z).

Like teleological explanations in the domain of human-made artefacts, certain teleological explanations in the biological domain are appropriate. For example, the teleological explanation "ears exist so that animals can hear" is appropriate, not because of intentional design, but because of the function-driven causal process of natural selection (Dennett, 2017; Lombrozo \& Carey, 2006; Wright, 1976). Hearing is a consequence of ears existing, and ears exist because the function of hearing meant those animals with ancient earlike structures out-reproduced animals that lacked these structures (Ayala, 2007; Dennett, 2017; Wright, 1976). However, natural selection is often misunderstood as an intentional, goaldirected process (Brumby, 1984). If the statement "ears exist so that animals can hear" were grounded in such a misunderstanding, this may imply that ears were designed so that animals can hear, and would constitute an inappropriate use of teleology from a scientific perspective (Roberts et al., 2020). Indeed, prior research has found that agreement with evolutionary teleological explanations is associated with a misunderstanding of the process of natural selection (Barnes et al., 2017). Therefore, while teleological explanations of biological adaptations can be appropriate, this is contingent upon an individuals' beliefs and knowledge about the process of natural selection. 
What both of Wright's (1976) formulations have in common, is that teleological explanations are causal. In the case of intentional action or design, if an agent wishes to achieve a certain outcome, and if the initiation of an action or the creation of an artefact is a way to reach that outcome, then teleology is clearly appropriate ${ }^{1}$. In the case of biological adaptations, if a biological trait serves a function, and if this function played a causal role in the selection of the trait through the non-intentional process of natural selection, then teleology is also appropriate.

However, other types of teleological explanation in the biological domain, such as "trees exist to produce oxygen for animals to breathe", are not appropriate, despite implicitly making similar causal claims. Animals being able to breathe is a consequence of trees producing oxygen, but trees do not produce oxygen because this results in animals being able to breathe. Teleological explanations in the nonbiological natural domain, such as "rivers flow downstream to get to the ocean", are not appropriate for the same reason. Getting to the ocean is a consequence of rivers flowing downstream, but rivers do not flow downstream because this results in getting to the ocean.

Despite teleological explanations of biological and nonbiological natural entities not being appropriate according to Wright's (1976) analysis, psychological research shows that such explanations are often judged to be true (Kelemen et al., 2013; Kelemen \& Rosset, 2009; Roberts et al., 2020, 2021). Given that the most ubiquitous use of teleology relates to the explanation of goal-directed behaviour or design by an intentional agent, and given that teleological beliefs involving biological adaptations often reflect a misbelief that natural

\footnotetext{
${ }^{1}$ Not all teleological explanations in the domains of intentional action and human-made artefacts are appropriate. For example, students do not attend medical school to go into debt. This is because although attending medical school tends to bring about debt, students do not attend medical school because it tends to bring about debt. Likewise, traffic lights do not change colour to use electricity. Although using electricity is a consequence of traffic lights changing colour, traffic lights were not designed to change colour because this results in the use of electricity. Therefore, although teleology may be appropriate in the domains of intentional action and human-made artefacts, this is partly contingent on the assumption of a particular outcome being a plausible goal for an agent.
} 
selection is goal-directed and intentional (Barnes et al., 2017), this raises the question of what underlies the acceptance of these "inappropriate" teleological explanations.

\section{Cognitive Accounts of Teleology}

Several psychological theories of teleological reasoning have been proposed. The first, known as selective teleology, posits that teleological explanations are elicited only by things which clearly possess some functional utility (Keil, 1992, 1995; see also Atran, 1995). According to this account, from a young age, children have a knowledge-acquisition device (or a "teleological-design stance") which predisposes them to favour teleological explanations of human-made artefacts and biological traits. Hence, teleology is said to be "selective" in the sense that even children should limit their use of teleological explanation to certain domains. Some support for this theory comes from findings that children prefer teleological explanations over mechanistic explanations for the properties of biological organisms, but prefer mechanistic explanations over teleological explanations for the properties of nonbiological natural entities (Keil, 1992). Children have also been found to ask more questions about the functions of human-made artefacts than of whole biological organisms, suggesting some level of domain specificity from an early age (Greif et al., 2006). Furthermore, although children can learn teleological explanations of natural nonbiological entities and generalise these explanations to novel entities, they are equally able to do this with mechanistic explanations (Lombrozo et al., 2018).

In contrast to the theory that teleological explanations are elicited only by things which possess some functional utility, the theory of promiscuous teleology posits that all teleological reasoning is psychologically based in an understanding that intentional agents have purposes (Kelemen, 1999a, 1999b, 2004). Evolutionary and modular theories of cognition (e.g., Atran \& Norenzayan, 2004; Boyer, 1994) often conceptualise a given cognitive process (e.g., facial recognition) as having a proper domain of stimuli that it evolved to respond to (i.e., faces), 
which is necessarily encompassed by an actual domain of stimuli that the cognitive process does respond to (i.e., faces, masks, and clouds with certain shapes). Similarly, Kelemen (1999a) argues that an early developing sensitivity to the intentions of agents is applied beyond its proper domains of intentional action and design. Like Wright (1976), Kelemen (1999a) suggests that all teleological explanations are implicitly causal, because regardless of whether teleology is appropriate, all teleological explanations reflect an implicit (mis)belief that an intention of a goal/function was the catalyst for a behaviour/existence of an object. However, where Wright's (1976) account is normative, Kelemen's (1999a) theory is descriptive. According to this theory, children, and to some extent, adults, should be accepting not only of teleological explanations about intentional action and human-made artefacts, but also of teleological explanations about biological and nonbiological natural entities.

Several lines of evidence support Kelemen's (1999a) theory. Compared to adults, young children are more accepting of teleological explanations about biological and nonbiological natural entities (Kelemen, 1999d, 1999c; Kelemen \& Diyanni, 2005), suggesting that in contrast to the predictions of Keil's $(1992,1995)$ theory, children are somewhat liberal in their use of teleological reasoning. In addition, under certain conditions, adults have also been shown to be accepting of teleological explanations for things which warrant only mechanistic explanations. Rates of teleological acceptance are higher in the absence of formal education (Casler \& Kelemen, 2008; Sánchez Tapia et al., 2016), when knowledge of alternative mechanistic explanations is impaired as a result of Alzheimer's disease (Lombrozo et al., 2007), or when individuals lack the time needed to inhibit a teleological response (Kelemen et al., 2013; Kelemen \& Rosset, 2009; Liquin \& Lombrozo, 2018 - study 5; Mills \& Frowley, 2014; Roberts et al., 2020). In conjunction with studies showing that acceptance of teleological explanations about biological and nonbiological natural entities is negatively related to the tendency to inhibit intuitions (Roberts et al., 2021; Zemla et al., 2012), these 
findings suggest that childlike teleological tendencies may persist throughout adulthood. Finally, in support of teleological reasoning being based in an understanding (and overattribution) of intentionality, studies have found positive relationships between acceptance of teleological explanations of biological and nonbiological natural entities and religious beliefs (Järnefelt et al., 2015; Kelemen \& Diyanni, 2005; Roberts et al., 2020, 2021), anthropomorphism (Roberts et al., 2021; Willard et al., 2020; Willard \& Norenzayan, 2013), and mother-nature beliefs (Kelemen et al., 2013; Liquin \& Lombrozo, 2018).

Recently, Liquin and Lombrozo (2018) proposed that teleological explanations tend to be more readily accepted when there is a high degree of perceived "fit" between a structure and a function. These authors found that by providing functional information in statements about biological entities, participants relied less on mechanistic details when assessing the statements. Importantly, when participants were asked to rate a series of teleological explanations about biological and nonbiological natural entities in terms of their perceived goodness of fit on a 7-point scale, the higher the perceived fit, the more likely the explanation was to be accepted as true. For every additional one-unit increase in ratings of fit, the odds of accepting the teleological explanation more than doubled. Liquin and Lombrozo (2018) argue that high structure-function fit provides a cue which makes some teleological explanations more compelling than others. This position is similar to the relational-deictic account proposed by Ojalehto et al. (2013). Specifically, the structure-function fit account and the relationaldeictic account both posit that the appropriateness of teleological explanation is not determined by anything intrinsic to the entity being explained, but rather, what determines whether teleological explanation is appropriate is the relationship between the entity and its environment.

Liquin and Lombrozo's (2018) structure-function fit account is also similar to Keil's $(1992,1995)$ theory that teleological reasoning is elicited by perceived functional utility, yet 
differs from both Keil (1992) and Kelemen (1999a), in the sense that it does not make domainspecific commitments about the origins of teleological reasoning. However, the idea that some teleological explanations are intuitively appealing is consistent with claims that teleological reasoning is a "developmentally persistent cognitive default" (Kelemen et al., 2013, p. 1075) originating from an early-developing understanding that intentional agents have purposes (Kelemen, 1999a). It is also consistent with evidence that adults display childlike teleological tendencies under certain circumstances (Casler \& Kelemen, 2008; Kelemen et al., 2013; Kelemen \& Rosset, 2009; Lombrozo et al., 2007; Mills \& Frowley, 2014; Roberts et al., 2020), and that acceptance of teleological explanations about biological and nonbiological natural entities is negatively related to the tendency to inhibit intuitions (Roberts et al., 2021; Zemla et al., 2012).

\section{Current Studies}

Liquin and Lombrozo's (2018) results raise several questions. Whereas Kelemen's (1999a) intention-based theory focuses on individual differences relating to teleology, Liquin and Lombrozo focused primarily on differences in teleological explanations themselves, which relate to whether a person accepts or rejects the explanation. Ratings of fit strongly predicted teleological acceptance at the level of the explanation itself, but it is not clear whether ratings of fit also vary at the individual-level. That is, do all individuals tend to view the same teleological explanations as having high (or low) structure-function fit, or do people vary in their perception of fit? If people vary in their perception of fit, this could contribute to individual differences in teleological acceptance. However, if people do not vary in their perception of fit, then this leads to the question of why, in the presence of high structurefunction fit, do some individuals accept the teleological explanation whereas others reject it?

Related to this point, it is unclear if, or how, the structure-function fit account may be compatible with the intention-based account, and indeed, whether both of these accounts could 
be consistent with Wright's (1976) analysis. According to Wright (1976), all teleological explanations are implicitly causal, and for a teleological explanation to be warranted, two criteria must be met. To borrow Liquin and Lombrozo's (2018) terminology, (1) the function would need to be a consequence of the structure being there, and (2) the structure would need to be there because it results in the function. By asking participants "to what extent do you think [explanandum] has a good fit with the function [function]?", it could be argued that Liquin and Lombrozo (2018) measured belief in something closely resembling Wright's (1976) first criterion. In support of this interpretation, Liquin and Lombrozo (2018) found that structure-function fit ratings also positively predicted teleological acceptance for explanations that are objectively true (e.g., "doctors prescribe antibiotics to treat infections") and those that are objectively false (e.g., "noses exist in order to support glasses"). False teleological control statements meet Wright's first, but not second criterion. Therefore, a positive relationship between structure-function fit ratings and acceptance of objectively false teleological explanations, should only be observed if structure-function fit measured belief that a function is a consequence of a structure being there, and not a belief that a structure is there because it results in a function.

As demonstrated using examples of teleological explanations in the domains of intentional behaviour and human-made artefacts, what satisfies Wright's (1976) second criterion is the fact that intentions are the catalyst of behaviour and design. Traffic lights change colour to control the flow of traffic, but traffic lights do not change colour to use electricity. Despite that traffic lights changing colour results in both the control of traffic and the use of electricity, only the first explanation is correct, because an intention to control the flow of traffic is the reason why traffic lights were designed to change colour. Applying this to the domains of biological and nonbiological natural entities, suppose "rivers flowing downstream" was judged to have a high fit with the function of "getting to the ocean". As 
Kelemen (1999a, 2004) argues that all teleological reasoning is psychologically based in an understanding of intentions, whether an individual then accepts the teleological explanation "rivers flow downstream to get to the ocean", may depend on whether they believe an intention to get to the ocean was the catalyst for flowing downstream. Prior research has established that teleological acceptance is positively predicted by belief in God (Järnefelt et al., 2015; Kelemen \& Diyanni, 2005) and anthropomorphism (Roberts et al., 2021; Willard et al., 2020; Willard \& Norenzayan, 2013), suggesting that teleological acceptance may be facilitated by an attribution of intentionality to an intending creator (belief in God) or to the entity itself (anthropomorphism). That is, high structure-function fit renders some teleological explanations more compelling than others (Liquin \& Lombrozo, 2018), but whether a person is receptive to this cue could depend on their tendency to believe that a structure exists because of a prior intention that a certain function occurs. In this way, Liquin and Lombrozo's (2018) theory of structure-function fit could be compatible with Kelemen's (1999a) intention-based theory, and both would be consistent with Wright's (1976) analysis.

\section{Study One}

Study One had three primary aims. First, we aimed to replicate findings by Liquin and Lombrozo (2018, study 5), which showed that ratings of structure-function fit positively predict acceptance of teleological explanations of biological and nonbiological natural entities. Second, we aimed to replicate findings of teleological acceptance being positively associated with anthropomorphism (Roberts et al., 2021; Willard et al., 2020; Willard \& Norenzayan, 2013) and negatively associated with the tendency to inhibit intuitions (Roberts et al., 2021; Zemla et al., 2012). Third, we sought to test whether the structure-function fit account (Liquin \& Lombrozo, 2018) is compatible with the intention-based account (Kelemen, 1999a, 1999c). Specifically, Study One explored whether anthropomorphism facilitates the transition from high structure-function fit to teleological acceptance, and whether a tendency to inhibit 
intuitions constrains the transition from high structure-function fit to teleological acceptance. The preregistration, data, and code for analyses for Study One are located on OSF (https://osf.io/wh7t3/?view_only=e8c8918ede3648558be6ff97ed1b967b).

\section{Method}

\section{Ethical Statement}

All studies in this manuscript were granted ethical clearance by the Macquarie University Human Research Ethics Committee under the project titled "Teleology and Structure-Function Fit” (protocol number 520211007828364).

\section{Participants}

An a-priori power analysis using the simr package in R (Green \& Macleod, 2016) revealed that a sample size of $N=125$ would provide power of $\sim 0.86$ for a small to medium effect $(d=0.30)$ for an interaction between anthropomorphism and structure-function fit. This sample size also gave adequate power to replicate previous findings of structure-function fit positively predicting teleological acceptance when participants were not under time pressure to respond (see pre-registration; and Liquin \& Lombrozo, 2018, study 5). One-hundred and twenty-five international participants were recruited through the online service, Prolific. Eligibility criteria included being a native English speaker and having normal or corrected-tonormal vision. To check that participants were carefully reading the questions, those who responded inaccurately to $20 \%$ or more of control statements on the Teleological Beliefs Scale were excluded from the analyses (e.g., Kelemen et al., 2013; Roberts et al., 2020, 2021). After exclusion, 114 participants remained. Ages for the final sample ranged from 18 to 71 ( $M$ $=33.46, S D=12.31$ ), with 46 males and 68 females. There were 23 agnostics, 30 atheists, one Buddhist, 40 Christians, one Hindu, one Jew, three Muslims, three Pagans, and 12 "other". 


\section{Materials}

Teleological Beliefs. To measure teleological beliefs about biological and nonbiological natural entities, participants responded to the Teleological Beliefs Scale (TBS: Roberts et al., 2021). The Teleological Beliefs Scale includes 98 statements across six categories to which participants respond "true" or "false". The six categories include biological teleological ( $n=14$; e.g., "Bacteria live in your gut in order to help with digestion"), nonbiological teleological ( $n=14$; e.g., "Rivers flow downstream in order to get to the ocean"), true teleological ( $n=10$; e.g., "Doctors prescribe antibiotics in order to treat infections"), false teleological ( $n=10$; e.g., "People buy food in order to spend money"), true causal ( $n=25$; e.g., "People earn money because they go to work"), and false causal ( $n=25$; e.g., "Saturn in a planet because it has sings surrounding it"). Responses to the biological and nonbiological teleological statements were of primary interest and were scored as either accepted or rejected (a response of true or false, respectively). As the statements in the other four categories were objectively true or objectively false, responses to these statements were scored as either accurate or inaccurate, and served as a control to ensure participants were paying attention. The 28 teleological statements of interest displayed excellent internal consistency $(\alpha=.91[.89$, $.94])$.

Structure-Function Fit. To measure the extent to which judgments of structurefunction fit predicted teleological acceptance, participants were presented with questions adapted from the 28 biological and nonbiological teleological statements from the Teleological Beliefs Scale. All structure-function fit questions were of the form "to what extent do you think [explanandum] has a good fit with the function [function]?" (e.g., "To what extent do you think that bacteria living in your gut has a good fit with the function of helping digestion?"). The content of every structure-function fit question corresponded to a particular statement from 
the Teleological Beliefs Scale. Each question was scored on a scale from 1 (not at all) to 7 (very much so).

Inhibition of Intuitions. Participants responded to a version of the seven-item Cognitive Reflections Test (CRT: Pennycook \& Rand, 2019). This includes re-worded versions of the original 3-item CRT (Frederick, 2005: e.g., "The ages of Mark and Adam add up to 28 years. If Mark is 20 years older than Adam, how many years old is Adam?"), as well as four less math-focused questions (Thomson \& Oppenheimer, 2016: e.g., "If you are running a race and you pass the person in second place, what place are you in?"). Each question has an intuitive, but incorrect answer. A total score is obtained by summing the number of correct responses. The internal consistency for the CRT was adequate $(\alpha=.67[.58, .77])$.

Anthropomorphism. Participants were presented with the Individual Differences in Anthropomorphism Questionnaire (IDAQ: Waytz et al., 2010). The IDAQ contains 15 items assessing the extent to which people attribute mental states (e.g., "To what extent does a tree have a mind of its own?"), free will (e.g., "To what extent does the average fish have free will?”), consciousness (e.g., “To what extent does a robot have consciousness?”), intentionality (e.g., "To what extent do cows have intentions?"), and emotion (e.g., "To what extent does a television set experience emotions?") to non-human entities. Each item is scored on a scale from 0 (not at all) to 10 (very much), and a total score is obtained by summing all 15 items. The IDAQ displayed good internal consistency $(\alpha=.82[.78, .87])$.

\section{Procedure}

Participants were directed to a Qualtrics-based questionnaire which took approximately 18 minutes to complete and were reimbursed $£ 2.25$ GBP for their time. The Teleological Beliefs Scale was presented first, followed by the Cognitive Reflection Test, structure-function fit questions, Individual Differences in Anthropomorphism Questionnaire, and finally, demographic questions. The presentation of items within each measure was fully randomised. 
Both the Teleological Beliefs Scale and structure-function fit questions automatically proceeded to the next item once a response had been recorded.

\section{Results}

\section{Descriptive Statistics}

In a preliminary exploration of our data, we computed teleological acceptance as the mean proportion of biological and nonbiological teleological statements accepted. As shown in Table 1, teleological acceptance was negatively related to the tendency to inhibit intuitions. Higher rates of teleological acceptance were positively related to anthropomorphism, but contrary to expectations, this was only marginally significant. Of particular interest, mean ratings of structure-function fit were not related to teleological acceptance, suggesting that structure-function fit is not sufficient for teleological acceptance.

\section{Table 1}

Correlations for Study One

\begin{tabular}{lcccc}
\hline & Teleology & Fit & IDAQ & CRT \\
\hline Teleology & - & & \\
& - & & \\
Fit & .02 & - & \\
& {$[-.16, .20]$} & -.08 & - \\
IDAQ & .17 & {$[-.26, .11]$} & - \\
& {$[-.01, .34]$} & -.01 & -.14 & - \\
CRT & $-.42 * * *$ & {$[-.19, .17]$} & {$[-.32, .05]$} & - \\
\hline
\end{tabular}

Note. Spearman correlations are shown with $95 \%$ confidence intervals [L, U] below. Teleology represents acceptance of items on the Teleological Beliefs Scale as a proportion. Fit represents average rating of structurefunction fit. IDAQ represents total score on the Individual Difference in Anthropomorphism Questionnaire. CRT represents accuracy on the extended Cognitive Reflections Test. $N=114$ for all cells.

$* * * p<.001$ 


\section{Analysis Plan}

Rather than calculating a mean teleological endorsement (e.g., Kelemen et al., 2013; Kelemen \& Rosset, 2009; Roberts et al., 2020, 2021), we instead used generalised linear mixed effects models with a logit function to test our hypotheses (Liquin \& Lombrozo, 2018) ${ }^{2}$. To allow for comparisons across models, we kept the random effect structure consistent throughout. For this reason, all models used random intercepts for participant and item, as the fixed effects in the models varied according to the specific hypothesis being tested. All predictors were mean-centred and z-transformed. Analyses were conducted in R (v.4.0.2) using the lme4 package (Bates et al., 2015).

\section{Replication of Prior Findings and Compatibility Between Accounts}

To replicate previous findings of ratings of structure-function fit positively predicting teleological acceptance (Liquin \& Lombrozo, 2018 - study 5), we ran a generalised linear mixed effects model with structure-function fit as a fixed effect and teleological acceptance as the outcome. As shown in Table 2 (Model 1), structure-function fit was a highly significant predictor of teleological acceptance, $\beta=1.39,95 \% C I[1.23,1.54], z=17.48, p<.001$. For every standard deviation increase in ratings of structure-function fit, the likelihood of teleological acceptance increased by $301 \%$.

The structure-function fit account and intention-based account are not necessarily incompatible in their claims. Next, we tested whether structure-function fit ratings and anthropomorphism have additive effects in explaining teleological acceptance, and indeed, whether the effect of structure-function fit is moderated by anthropomorphism. As shown in Table 2 (Model 2), structure-function fit, $\beta=1.40,95 \% C I[1.25,1.55], z=17.55, p<.001$, and anthropomorphism, $\beta=0.40,95 \% C I[0.04,0.76], z=2.16, p=.031$, were both significant

\footnotetext{
${ }^{2}$ The analyses reported here differ slightly from the pre-registration. The reason for this was to ensure consistency with the pre-registered analyses of Study Three. However, all pre-registered analyses from Study One are included in the supplementary materials. The conclusions do not change.
} 
predictors of teleological acceptance. However, these main effects were qualified by a significant interaction, $\beta=0.14,95 \% C I[0.01,0.27], z=2.12, p=.034$. This was such that the increase in likelihood of teleological acceptance for every standard deviation increase in structure-function fit (306\%), increased by a further $15 \%$ for every standard deviation increase in anthropomorphism.

\section{Table 2}

Generalised Linear Mixed Effects Models Predicting Teleological Acceptance in Study One

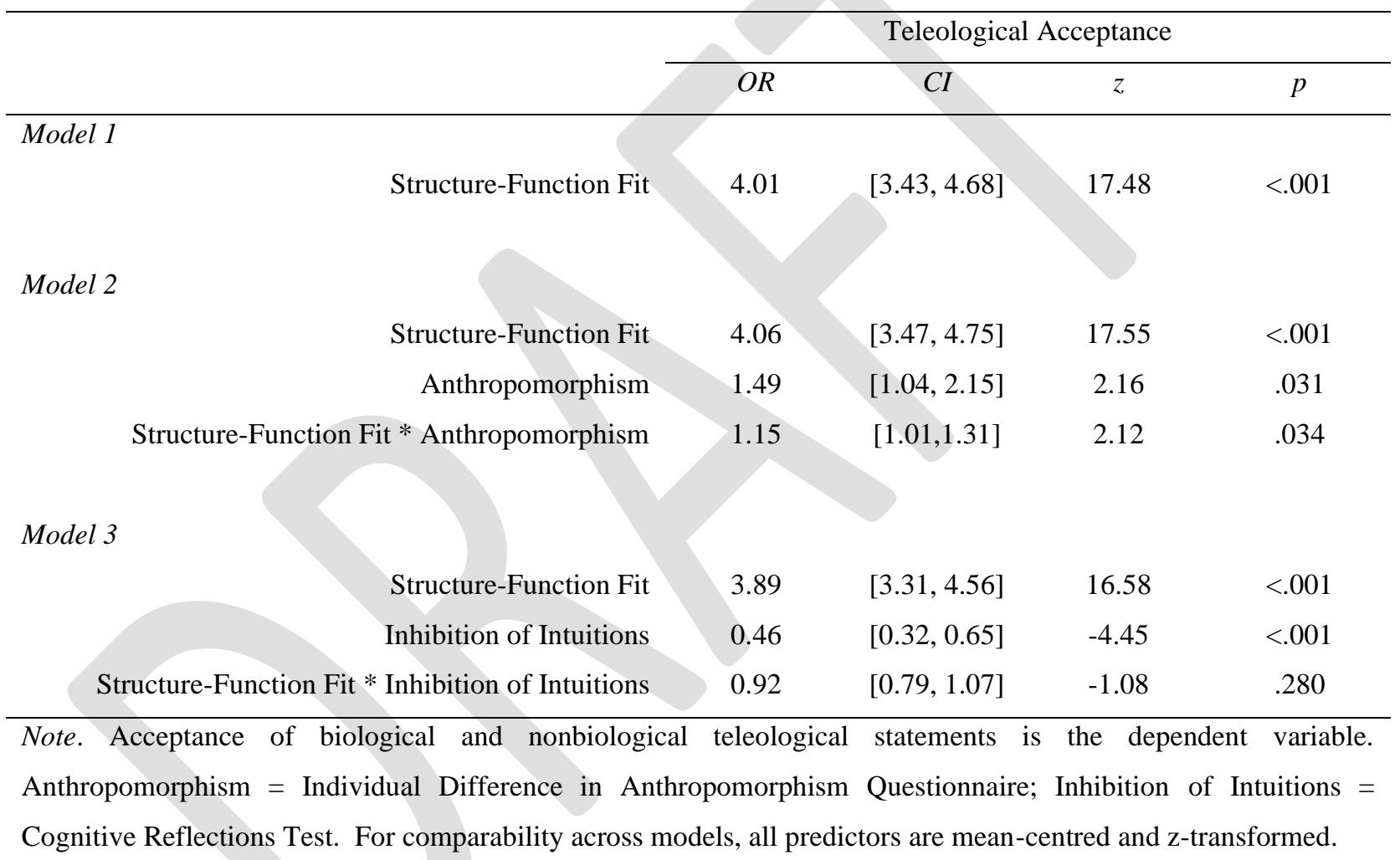

Finally, we explored whether structure-function fit ratings and the tendency to inhibit intuitions have additive effects in explaining teleological acceptance, and whether the effect of structure-function fit is moderated by the tendency to inhibit intuitions. As shown in Table 2 (Model 3), structure-function fit, $\beta=1.36,95 \% C I[1.25,1.56], z=16.58, p<.001$, and inhibition of intuitions, $\beta=-0.78,95 \% C I[-1.13,-0.44], z=-4.45, p<.001$, were both significant predictors of teleological acceptance. For every standard deviation increase in 
structure-function fit, the likelihood of teleological acceptance increased by $288 \%$, whereas for every standard deviation increase in inhibition of intuitions, the likelihood of teleological acceptance decreased by $54 \%$. Contrary to expectations, the effect of structure-function fit was not moderated by inhibition of intuitions, $\beta=-0.09,95 \% C I[-0.24,0.07], z=-1.08, p=.280$.

\section{Discussion}

The results of Study One replicated those of Liquin and Lombrozo (2018, study 5), in showing that perceived goodness of fit was a highly significant positive predictor of teleological acceptance. Consistent with previous findings of teleological acceptance being negatively related to the tendency to inhibit intuitions (Roberts et al., 2021; Zemla et al., 2012), scores on the CRT negatively predicted teleological acceptance in both the bivariate correlations and generalised linear mixed effects model. However, in contrast to previous findings of teleological acceptance being positively related to anthropomorphism (Roberts et al., 2021; Willard et al., 2020; Willard \& Norenzayan, 2013), the current findings were mixed. Bivariate correlations showed no significant relationship between teleological acceptance and anthropomorphism, yet the generalised linear mixed effects model revealed a significant relationship in the expected direction. Although structure-function fit ratings were highly predictive of whether a teleological explanation is seen as appropriate, examination of the bivariate correlations showed that individual differences in perceived goodness of fit were not related to individual differences in teleological acceptance. While high perceived fit may provide a cue that a particular teleological explanation is appropriate, individuals who tended to provide judgements of higher fit were no more accepting of teleological explanations. This suggests that perceived goodness of fit may be necessary but not sufficient for teleological acceptance.

As the structure-function fit account holds that goodness of fit may act as a cue that a teleological explanation is appropriate - meaning that certain teleological explanations should 
be more compelling than others - this leads to the prediction that the effect of structure-function fit may depend on individual differences such as the tendency to inhibit intuitions and to anthropomorphise. Although Study One found no evidence that the effect of structure-function fit was weaker for individuals with a tendency to inhibit their intuitions, we did find that the effect of structure-function fit was stronger for highly anthropomorphic individuals. This suggests that highly anthropomorphic individuals may find high structure-function fit an especially compelling cue that teleological explanation is appropriate. However, these results do not speak to the issue of what people interpret "fit" to mean.

\section{Study Two}

In Study Two, we investigate whether questions about structure-function fit are interpreted as asking about a causal relationship between a structure and function. To achieve this aim, we calculated two measures of causal beliefs from conditional statements of the forms "if $p$, then $q$ " and "if not $p$, then $q$ ". Probability of the conditional was calculated as agreement with the first type of conditional, and delta-p was calculated as agreement with the first type of conditional minus agreement with the second type of conditional (Cheng, 1997; Evans et al., 2007; Over, 2017; Over et al., 2007). These correspond to belief in whether an outcome will occur following a specific event, and whether an outcome will only occur following a specific event, respectively.

The reason for thinking people may interpret questions about structure-function fit as asking about causality, is that according to Wright's (1976) first criterion, a factor in determining if teleology is appropriate in this context would be whether a structure (or entity) tends to bring about a function (or outcome). In cases where this first criterion is met, this would not necessarily mean that teleology is appropriate, as the second criterion would also have to be met. For example, rain falling may tend to bring about plants growing, but unless plants growing is also the reason why rain falling occurs, then a strong causal relationship 
would not be sufficient for teleological acceptance. Relating this to the results of Study One, this would explain why ratings of fit predicted teleological acceptance at the item-level, but not at the individual-level. Teleology may be seen as more appropriate when there is a strong causal relationship between a structure and function (i.e., high fit) compared to when there is a weak causal relationship between a structure and function (i.e., low fit). However, whether an individual makes the leap to teleological acceptance would also depend on whether they view the function as having played a causal role in bringing about the structure (Lombrozo \& Carey, 2006; Wright, 1976).

\section{Method}

\section{Participants}

An a-priori power analysis using the simr package in R (Green \& Macleod, 2016) revealed that a sample size of $N=100$ would provide power of $\sim 0.90$ for a small to medium effect $(d=0.30)$ of delta-p on ratings of structure-function fit after controlling for probability of the conditional. One-hundred and two native English speakers were recruited online through Prolific. Three participants were excluded for incomplete responses. Ages for the final sample $(N=99)$ ranged from 18 to $88(M=30.83, S D=13.74)$, with 47 males, 50 females, and two non-binary.

\section{Materials}

Structure-Function Fit. Participants responded to the same 28 structure-function fit questions about biological and nonbiological natural entities as in Study One. In addition, participants responded to ten structure-function fit questions adapted from the false teleological control statements in the Teleological Beliefs Scale (e.g., "To what extent do you think that noses have a good fit with the function of supporting glasses?"). These statements were included primarily to encourage participants to use the full range of response options, from 1 (not at all) to 7 (very much so). All false teleological control statements meet Wright's (1976) 
first criterion of the function occurring as a consequence of the structure, but are false by virtue of not meeting Wright's second criterion of the structure being there because it results in the function. Therefore, this also enabled the dissociation of the two criteria in terms of how people interpret questions about structure-function fit.

Causal Beliefs. The content of each structure-function fit question was paired with two conditional statements: one of the form if $p$, then $q$ (e.g., "If rain falls, then plants will grow"), and one of the form if not p, then $q$ (e.g., "If rain does not fall, then plants will grow"). Participants were asked to rate how likely they thought each statement was to be true, on a scale from 0 (definitely not true) to 10 (definitely true). From this, we calculated two measures of belief. Probability of the conditional was calculated as the rating of statements of the form if $p$, then $q$. Probability of the conditional could therefore range from 0 to 10 , with higher scores indicating a stronger belief that a given effect would occur in the presence of a cause. Delta-p was calculated as the rating of statements of the form if $p$, then $q$, minus the ratings of statements of the form if not $p$, then $q$. Delta-p could therefore range from -10 to 10 , with positive scores indicating belief that an effect was more likely to occur in the presence rather than absence of a certain cause, and negative scores indicating a belief that an effect was more likely to occur in the absence rather than presence of a certain cause. ${ }^{3}$

\section{Procedure}

Participants were directed to a Qualtrics-based questionnaire which took approximately 12 minutes to complete and were reimbursed $£ 1.50 \mathrm{GBP}$ for their time. The structure-function fit questions were presented first, followed by the conditional statements, and finally, demographic questions. The presentation of items within each measure was fully randomised. Both the structure-function fit questions and conditional statements automatically proceeded

\footnotetext{
${ }^{3}$ Note that in cases where an effect was judged to have two or more orthogonal causes, probability of the conditional and delta-p should differ.
} 
to the next item once a response had been recorded. The pre-registration for Study Two is located on OSF (https://osf.io/sb89j/?view_only=d680d9a1f6604fa5954d873acb272971).

\section{Results}

\section{Descriptive Statistics}

Questions about biological and nonbiological natural entities were rated as having higher structure-function fit $(M=5.65, S D=0.75)$ than questions adapted from false teleological control statements $(M=4.75, S D=0.97), t(98)=3.30, p=.002, d=0.331$. In terms of causal beliefs, delta-p was significantly higher for content referring to biological and nonbiological natural entities $(M=5.78, S D=1.85)$ compared to content adapted from false teleological controls $(M=3.82, S D=2.14), t(98)=3.62, p=.001, d=.364$, whereas probability of the conditional did not differ significantly between content referring to biological and nonbiological natural entities $(M=7.99, S D=1.09)$ and content adapted from false teleological controls $(M=7.47, S D=1.11), t(98)=1.53, p=.136, d=0.153$.

False teleological control statements (e.g., "noses exist in order to support glasses") meet Wright's (1976) first criterion of the function being a consequence of the structure being there, but are "false" because they do not meet Wright's second criterion of the structure being there because it results in the function. Such statements tend to be accepted at close to floor levels (Roberts et al., 2021). Therefore, if structure-function fit measured belief in Wright's second criterion, ratings of fit for these statements should also be close to floor level. A onesample t-test showed that content adapted from false teleological control statements did differ significantly from floor level in ratings of structure-function fit, $t(98)=38.36, p<.001, d=$ 3.866. Furthermore, false teleological control statements differed significantly from floor level in ratings of probability of the conditional, $t(98)=66.81, p<.001, d=6.730$, and delta-p, $t(98)$ $=17.76, p<.001, d=1.785$, indicating that structure-function fit, probability of the conditional, and delta-p, all measure belief in Wright's first, rather than second criterion. 
Bivariate correlations (Table 3) revealed that for statements about biological and nonbiological natural entities, ratings of structure-function fit were strongly, positively, and significantly related to both probability of the conditional and delta-p. For statements adapted from false teleological controls, ratings of fit displayed moderate-to-strong significant positive relationships with probability of the conditional and delta-p. For both types of content, probability of the conditional and delta-p were strongly, positively, and significantly related to one another.

Table 3

Correlations Between Structure-Function Fit and Causal Beliefs in Study Two



$* * * p<.001, * * p<.01$

\section{Structure-Function Fit and Responses to Conditionals}

To explore the relative effects of probability of the conditional and delta-p on ratings of structure-function fit, we ran two linear mixed effects models: one for content about biological and nonbiological natural entities, and one for content about false teleological 
controls. Both models included random intercepts for participant and item. As probability of the conditional and delta-p were measured on different scales, both were z-transformed. As shown in Table 4, probability of the conditional and delta-p were both significant predictors of ratings of fit for questions about biological and nonbiological natural entities. However, only probability of the conditional was a significant predictor of fit for questions about false teleological controls.

Table 4

Linear Mixed Effects Models Predicting Fit from Probability of Conditional and Delta-P

\begin{tabular}{lcccccc}
\hline & \multicolumn{2}{c}{ Teleological Test } & \multicolumn{2}{c}{ False Teleological } \\
\hline & Beta & $t$ & $p$ & Beta & $t$ & $p$ \\
\hline Probability Conditional & $0.46[0.38,0.54]$ & 11.77 & $<.001$ & $0.47[0.35,0.59]$ & 7.63 & $<.001$ \\
Delta-P & $0.18[0.11,0.26]$ & 4.67 & $<.001$ & $0.10[-0.04,0.23]$ & 1.43 & .154 \\
\hline
\end{tabular}

Note. Both models use random intercepts of participant and item. Probability of the conditional and delta-p are both z-transformed.

\section{Discussion}

As discussed earlier, all false teleological control statements meet Wright's (1976) first criterion of the function occurring as a consequence of the structure, but are false by virtue of not meeting the second criterion of the structure being there because it results in the function. As probability of the conditional was a significant predictor of structure-function fit ratings for these false teleological statements, the results of Study Two suggest that questions about structure-function fit may be interpreted as asking whether a structure plays a causal role in bringing about a function, rather than whether the structure is there because it results in the function. In other words, structure-function fit appears to measure belief in Wright's first criterion rather than the second criterion.

Of the two measures of causal belief, probability of the conditional was a stronger predictor of ratings of fit than delta-p was. Although both measures have been shown to relate 
to explicit judgements of causality (i.e., whether p causes $\mathrm{q}$ ), prior work has also found that probability of the conditional is the stronger of the two (Over et al., 2007). Furthermore, while both are related to explicit judgements of causality, probability of the conditional and delta-p arguably measure aspects of causal beliefs. Positive scores for probability of the conditional are related to belief that $\mathrm{q}$ will occur given $\mathrm{p}$, whereas positive scores for delta-p are related to belief that q will only occur given $\mathrm{p}$. These results suggest that structure-function fit is more closely related to belief that a function will occur given a structure, than to a belief that a function will only occur given a structure.

\section{Study Three}

Study Two showed that ratings of structure-function fit are closely related to the belief that a function will occur given the presence of a structure. While this may be necessary for teleological acceptance, the results of Study One, in combination with Wright's (1976) analysis, suggest this is not sufficient for teleological acceptance. What should be necessary and sufficient for teleological acceptance is the belief that a function will occur in the presence of a structure, and the belief that the structure exists because it tends to result in the function. Indeed, previously reported findings on rates of acceptance for teleological explanations about biological and nonbiological natural entities compared to rates acceptance for teleological explanations that are objectively false by virtue of not satisfying Wright's (1976) second criterion, support this argument. Specifically, whereas teleological explanations about biological and nonbiological natural entities tend to be accepted at moderate rates, false teleological explanations tend to be accepted close to floor levels (Roberts et al., 2021). The importance of this second criterion for acceptance of teleological explanations has previously been demonstrated in the domains of human-made artefacts and artificial selection (Lombrozo \& Carey, 2006). Therefore, while high structure-function fit (i.e., the belief that a function will 
occur in the presence of a structure) may be a salient cue that teleological explanation could be appropriate, this does not guarantee teleological acceptance (Liquin \& Lombrozo, 2018).

In the context of intentional action and human-design, Wright's (1976) second criterion is met because intentions are the catalyst of both purposeful behaviour and the creation of artefacts. According to Kelemen (1999a, 2004), all teleological reasoning is psychologically based in an understanding that intentional agents have purposes. The previously reported positive relationships between acceptance of teleological explanations about biological and nonbiological natural entities and belief in God (Järnefelt et al., 2015; Kelemen \& Diyanni, 2005; Roberts et al., 2020, 2021) and anthropomorphism (Roberts et al., 2021; Willard et al., 2020; Willard \& Norenzayan, 2013), suggest that attributions of intentionality may also satisfy Wright's (1976) second criterion in the current context. Therefore, Study Three aimed to determine whether attributions of intentionality facilitate the leap from a strong causal relationship to teleological acceptance. Specifically, we investigate whether individuals who are highly anthropomorphic or express greater belief in God are more likely to view a strong causal relationship as appropriate for teleological explanation.

Due to the ease with which we attribute intentions to other individuals, Kelemen et al. (2013, p. 1075) claim that teleological reasoning represents a "developmentally persistent cognitive default". Liquin and Lombrozo (2018) take a slightly more nuanced view, in claiming that only certain teleological explanations are highly compelling due to their "goodness of fit". The negative relationship between teleological acceptance and the tendency to inhibit intuitions (Roberts et al., 2021; Zemla et al., 2012), as well as reports of increased teleological acceptance when under time pressure to respond (Kelemen et al., 2013; Kelemen \& Rosset, 2009; Liquin \& Lombrozo, 2018; Mills \& Frowley, 2014; Roberts et al., 2020), suggest that at least some teleological explanations about biological and nonbiological natural entities are intuitively appealing. However, if the best candidates for teleological explanation 
are those in which there is a strong causal link between a structure and function, then these are the ones in which inhibiting intuitions should be necessary if an individual is to judge the explanation as false. Therefore, Study Three also aimed to determine whether the tendency to inhibit intuitions constrains the effect of a strong causal link between a structure and a function on teleological acceptance. In this way, Study Three aimed to expand on previous findings from both the structure-function fit (Liquin \& Lombrozo, 2018) and intention-based (Kelemen, 1999a; Kelemen et al., 2013; Kelemen \& Rosset, 2009; Roberts et al., 2020; Zemla et al., 2012) accounts of teleology to determine whether the two accounts are compatible, and indeed, whether both are consistent with Wright's (1976) analysis.

\section{Methods}

\section{Participants}

An a-priori power analysis using the simr package in R (Green \& Macleod, 2016) revealed that a sample size of $N=250$ would provide power of $\sim 0.82$ to detect a small effect $(d=0.20)$ for an interaction between causal beliefs and anthropomorphism in predicting teleological acceptance. Two-hundred and fifty native English speakers were recruited online through Prolific. To ensure that religious beliefs did not involve belief in the intrinsic agency of nature, only those identifying as non-religious or from an Abrahamic affiliation were eligible to participate (Roberts et al., 2021). One participant was excluded for not completing the entire study, nine for indicating they held religious beliefs that did not align with the selection criteria, and 23 for failing to correctly respond to over $80 \%$ of control statements. After exclusion, 217 participants remained. Ages for the final sample ranged from 18 to $64(M=30.05, S D=9.97)$, with 65 males, 150 females, one other, and one who preferred not to say. Forty-eight participants identified as agnostic, 72 as atheist, 91 as Christian, one as Jewish, and five as Muslim. 


\section{Materials}

Most materials in Study Three were identical to those used in the previous studies. To measure teleological beliefs about biological and nonbiological natural entities, participants responded to the Teleological Beliefs Scale (Roberts et al., 2021). Causal beliefs were measured with the same conditional statements as those used in Study Two, from which probability of the conditional and delta-p were calculated. Anthropomorphism was measured with the Individual Differences in Anthropomorphism Questionnaire (Waytz et al., 2010), and the tendency to inhibit intuitions was measured with the extended version of the Cognitive Reflection Test (Frederick, 2005; Thomson \& Oppenheimer, 2016).

To measure religiosity, we presented participants with the Centrality of Religiosity Scale (CRS: Huber \& Huber, 2012). The CRS contains 15 items across five subscales: experience (e.g., "How often do you experience situations in which you have the feeling that God or something divine intervenes in your life?"), ideology (e.g., "In your opinion, how probable is it that a higher power really exists?"), intellect (e.g., "How interested are you in learning more about religious topics?"), private practise (e.g., "How important is personal prayer to you?"), and public practise (e.g., "How often do you take part in religious services?”). However, as only the experience, ideology, and private practise subscales necessarily relate to a belief in God (Roberts et al., 2021), in the pre-registration we restricted analyses to a reduced version of the CRS containing only these subscales ${ }^{4}$. Two questions about the objective frequency of private prayer were scored on a scale from 1 (never) to 8 (several times a day), and were then re-coded to a five-point scale according to Huber and Huber (2012). The remaining seven items were scored on a scale from 1 (never/not at all) to 5 (very often/very much so). A total score was obtained by taking the mean of all nine items, such that higher scores are indicative of a greater belief in God.

\footnotetext{
${ }^{4}$ See the supplementary materials for analyses using the full scale. Interpretation of the results does not change.
} 


\section{Procedure}

Participants were directed to a Qualtrics-based questionnaire which took approximately 25 minutes to complete and were reimbursed $£ 3.13$ GBP for their time. The Teleological Beliefs Scale was presented first, followed by the Cognitive Reflection Test. The Individual Differences in Anthropomorphism Questionnaire and Centrality of Religiosity Scale were presented next in random order, followed by the conditional statements, and finally, demographic questions. The presentation of items within each measure was fully randomised. Both the Teleological Beliefs Scale and conditional statements automatically proceeded to the next item once a response had been recorded. The pre-registration for Study Three is located on OSF (https://osf.io/avmbh/?view_only=af57311ad7284766bc0255e9ba130118).

\section{Results}

\section{Descriptive Statistics}

Bivariate correlations revealed a pattern of results similar to Study One. As shown in Table 5, individuals who rated causal strength as higher were not any more accepting of teleological explanations on average. Rates of acceptance for false teleological controls displayed the same pattern of results: individuals who tended to rate causal strength as higher were not any more accepting of the false teleological controls on average. However, there was a moderate-to-strong positive significant relationship between acceptance of teleological explanations about biological and nonbiological natural entities and acceptance of false teleological explanations.

Replicating previous findings, rates of teleological acceptance were significantly and positively correlated with anthropomorphism and belief in God (Roberts et al., 2021; Willard et al., 2020; Willard \& Norenzayan, 2013), and significantly and negatively correlated with the tendency to inhibit intuitions (Roberts et al., 2021; Zemla et al., 2012). In contrast, rates of acceptance for false teleological controls were not significantly correlated with 
anthropomorphism or belief in God, and only weakly and negatively correlated with the tendency to inhibit intuitions. 
Table 5

Study Three Correlations

\begin{tabular}{|c|c|c|c|c|c|c|c|c|c|}
\hline & Test Acc & Test PoC & Test D-P & False Acc & False PoC & False D-P & IDAQ & r-CRS & CRT \\
\hline Test Acc & - & & & & & & & & \\
\hline Test PoC & $\begin{array}{c}.04 \\
{[-.09, .17]}\end{array}$ & - & & & & & & & \\
\hline Test D-P & $\begin{array}{c}.06 \\
{[-.07, .19]}\end{array}$ & $\begin{array}{c}.85 * * * \\
{[.81, .88]}\end{array}$ & - & & & & & & \\
\hline False Acc & $\begin{array}{c}.43 * * * \\
{[.31, .53]}\end{array}$ & $\begin{array}{c}-.11 \\
{[-.24, .02]}\end{array}$ & $\begin{array}{c}-.06 \\
{[-.19, .07]}\end{array}$ & & & & & & \\
\hline False PoC & $\begin{array}{c}.11 \\
{[-.24, .03]}\end{array}$ & $\begin{array}{c}.67 * * * \\
{[.59, .73]}\end{array}$ & $\begin{array}{c}.57 * * * \\
{[.47, .65]}\end{array}$ & {$[-.14, .12]$} & & & & & \\
\hline False D-P & $\begin{array}{c}.03 \\
{[-.11, .16]}\end{array}$ & $\begin{array}{c}.47 * * * \\
{[.36, .57]}\end{array}$ & $\begin{array}{c}.61 * * * \\
{[.51, .68]}\end{array}$ & $\begin{array}{c}.09 \\
{[-.04, .22]}\end{array}$ & & - & & & \\
\hline IDAQ & $\begin{array}{c}.37 * * * \\
{[.25, .48]}\end{array}$ & $\begin{array}{c}-.02 \\
{[-.15, .11]}\end{array}$ & $\begin{array}{c}-.09 \\
{[-.22, .04]}\end{array}$ & $\begin{array}{c}.13 \\
{[-.01, .26]}\end{array}$ & $\begin{array}{c}-.03 \\
{[-.17, .10]}\end{array}$ & $\begin{array}{c}-.05 \\
{[-.19, .08]}\end{array}$ & - & & \\
\hline $\mathrm{r}-\mathrm{CRS}$ & $\begin{array}{c}.37 * * * \\
{[.25, .48]}\end{array}$ & $\begin{array}{c}-.06 \\
{[-.19, .07]}\end{array}$ & $\begin{array}{c}-.01 \\
{[-.14, .12]}\end{array}$ & $\begin{array}{c}.12 \\
{[-.01, .25]}\end{array}$ & $\begin{array}{c}-.12 \\
{[-.25, .02]}\end{array}$ & $\begin{array}{c}-.01 \\
{[-.15, .12]}\end{array}$ & $\begin{array}{c}.17^{*} \\
{[.04, .30]}\end{array}$ & - & \\
\hline CRT & $\begin{array}{c}-.44 * * * \\
{[-.54,-.33]}\end{array}$ & $\begin{array}{c}-.09 \\
{[-.22, .04]}\end{array}$ & $\begin{array}{c}-.14^{*} \\
{[-.27,-.01]}\end{array}$ & $\begin{array}{c}-.14^{*} \\
{[-.27,-.004]}\end{array}$ & $\begin{array}{c}.01 \\
{[-.12, .15]}\end{array}$ & $\begin{array}{c}-.20 * * \\
{[-.33,-.07]}\end{array}$ & $\begin{array}{c}-.09 \\
{[-.22, .04]}\end{array}$ & $\begin{array}{c}-.28 * * * \\
{[-.40,-.15]}\end{array}$ & - \\
\hline
\end{tabular}

Note. Pearson's r shown with 95\% CI [L, U]. Test = teleological statements about biological and nonbiological natural entities, False $=$ false teleological control statements, Acc $=$ acceptance of statements, PoC $=$ probability of the conditional, D-P = delta-p, IDAQ $=$ Individual Differences in Anthropomorphism Questionnaire, $\mathrm{r}-\mathrm{CRS}=$ reduced Centrality of Religiosity Scale, CRT $=$ Cognitive Reflection Test. $N=217$ for all cells.

$* p<.05 ; * * p<.01 ; * * * p<.001$ 


\section{Causal Beliefs and Individual Differences}

To investigate whether teleological explanations were more readily accepted when they were judged to have strong causal relationships, and whether the effect of causality was moderated by individual differences in anthropomorphism, belief in God, and inhibition of intuitions, we ran a series generalised linear mixed effects models with logit functions. All models included random intercepts for participants and items. As the predictors were measured on different scales, and due to the predicted interactions, all predictors were z-transformed and mean-centred ${ }^{5}$.

We first investigated whether statements with higher ratings of causality were more likely to be accepted in their teleological form. As shown in Table 6 (Model 1), for every standard deviation increase in probability of the conditional, the likelihood of acceptance for teleological explanations about biological and nonbiological natural entities increased by $93 \%$. Similarly, for every standard deviation increase in probability of the conditional, the likelihood of acceptance for false teleological explanations increased by $57 \%$.

\footnotetext{
${ }^{5}$ As Study Two revealed probability of the conditional was a stronger predictor than delta-p of structurefunction fit, here we focus our interpretation of results primarily on the former rather than the latter. However, all models were repeated using delta-p as the measure of causal belief and the interpretation does not change. See Supplementary Materials (Table S2).
} 
Table 6

Generalised Linear Mixed Effects Models Predicting Teleological Acceptance in Study Three

\begin{tabular}{|c|c|c|c|c|c|c|c|c|}
\hline & \multicolumn{4}{|c|}{ Biological and Nonbiological } & \multicolumn{4}{|c|}{ False Control } \\
\hline & $O R$ & $C I$ & $z$ & $p$ & $O R$ & $C I$ & $z$ & $p$ \\
\hline \multicolumn{9}{|l|}{ Model 1} \\
\hline Causality & 1.93 & {$[1.77,2.12]$} & 14.41 & $<.001$ & 1.57 & {$[1.15,2.13]$} & 2.88 & .004 \\
\hline \multicolumn{9}{|l|}{ Model 2} \\
\hline Causality & 1.93 & {$[1.76,2.11]$} & 14.38 & $<.001$ & 1.64 & {$[1.19,2.27]$} & 3.03 & .002 \\
\hline Anthropomorphism & 1.87 & {$[1.51,2.31]$} & 5.79 & $<.001$ & 1.38 & {$[1.03,1.85]$} & 2.14 & .033 \\
\hline Causality * Anthropomorphism & 1.08 & {$[1.002,1.17]$} & 2.02 & .044 & 0.84 & {$[0.64,1.09]$} & -1.30 & .195 \\
\hline \multicolumn{9}{|l|}{ Model 3} \\
\hline Causality & 1.87 & {$[1.70,2.04]$} & 13.36 & $<.001$ & 1.58 & {$[1.19,2.27]$} & 2.81 & .005 \\
\hline Belief in God & 1.91 & {$[1.55,2.35]$} & 6.02 & $<.001$ & 1.38 & {$[1.03,1.85]$} & 2.09 & .037 \\
\hline Causality * Belief in God & 1.16 & {$[1.07$} & 6 & $<.001$ & 1.02 & {$[0.64,1.09]$} & 0.13 & .894 \\
\hline \multicolumn{9}{|l|}{ Model 4} \\
\hline Causality & 1.87 & {$[1.71,2.05]$} & 13.51 & $<.001$ & 1.48 & {$[1.08,2.01]$} & 2.45 & .014 \\
\hline Inhibition & 0.49 & {$[0.40,0.60]$} & -6.80 & $<.001$ & 0.78 & {$[0.58,1.05]$} & -1.62 & .106 \\
\hline Causality $*$ Inhibition & 0.89 & {$[0.82,0.97]$} & -2.67 & .008 & 0.84 & {$[0.63,1.10]$} & -1.27 & .204 \\
\hline
\end{tabular}

Note. Generalised linear mixed effects models predicting acceptance of teleological statements about biological and nonbiological natural entities (left) and false control statements (right). "Causality" = probability of the conditional, "Anthropomorphism" = IDAQ total score, "Belief in God" = reduced CRS mean score, "Inhibition" = CRT total score. For interpretability of the interactions, all predictors are mean-centred and z-transformed. 
Next, we investigated whether anthropomorphism facilitates teleological acceptance in the presence of a strong causal relationship. As shown in Table 6 (Model 2), probability of the conditional and anthropomorphism both positively predicted acceptance of teleological explanations about biological and nonbiological natural entities. As predicted, there was a significant interaction between probability of the conditional and anthropomorphism, showing that as anthropomorphism increased, the effect of causality on teleological acceptance increased (see Figure 1). The likelihood of acceptance for teleological explanations about biological and nonbiological natural entities associated with a standard deviation increase in probability of the conditional (93\%), increased by a further $8 \%$ for every standard deviation increase in anthropomorphism. Probability of the conditional and anthropomorphism also positively predicted acceptance of false teleological control statements. However, in contrast to explanations about biological and nonbiological natural entities, anthropomorphism did not facilitate acceptance of false teleological control statements in the presence of a strong causal relationship.

Figure 1. Teleological Acceptance as a Function of Causality and Anthropomorphism

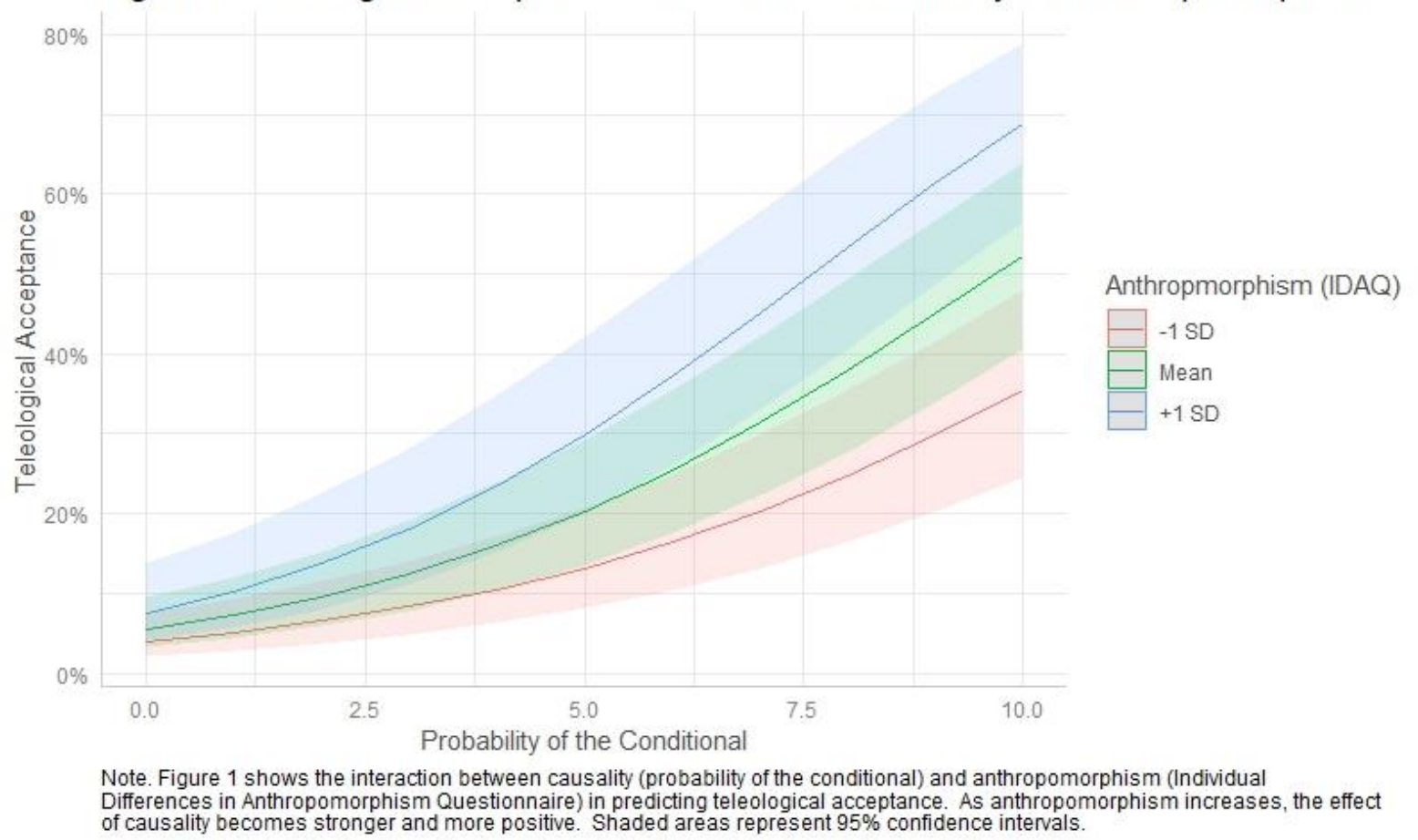


Next, we investigated whether belief in God facilitates teleological acceptance in the presence of a strong causal relationship. As shown in Table 6 (Model 3), probability of the conditional and belief in God both positively predicted acceptance of teleological explanations about biological and nonbiological natural entities. As predicted, there was a significant interaction between probability of the conditional and belief in God, showing that as belief in God increased, the effect of causality on teleological acceptance increased (see Figure 2). The likelihood of acceptance for teleological explanations about biological and nonbiological natural entities associated with a standard deviation increase in probability of the conditional $(87 \%)$, increased by a further $16 \%$ for every standard deviation increase in belief in God. Probability of the conditional and belief in God also positively predicted acceptance of false teleological control statements. However, in contrast to explanations about biological and nonbiological natural entities, belief in God did not facilitate acceptance of false teleological control statements in the presence of a strong causal relationship.

Figure 2. Teleological Acceptance as a Function of Causality and Belief in God

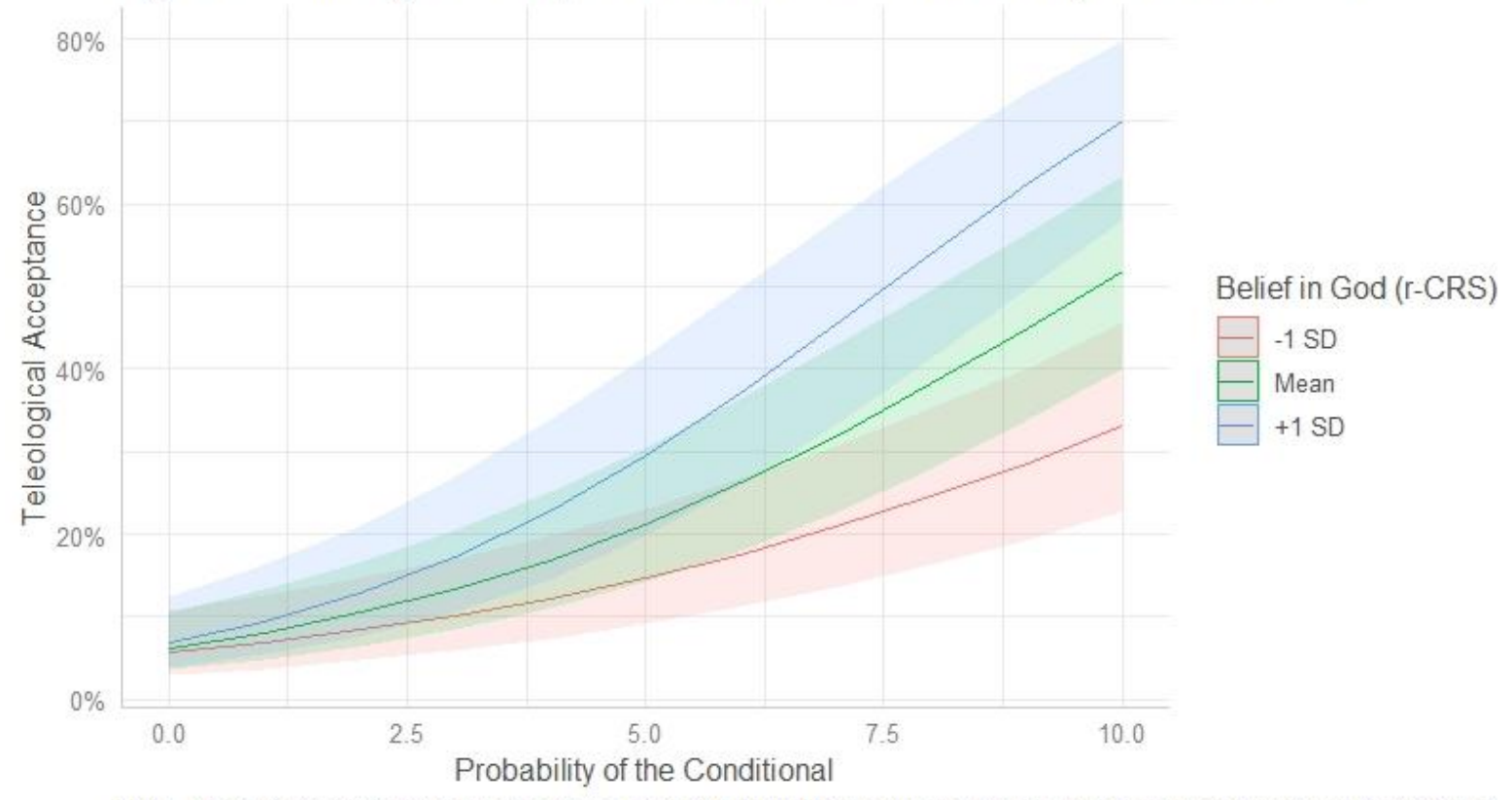

Note. Figure 2 shows the interaction between causality (probability of the conditional) and belief in God (reduced Centrality of Religiosity Scale) in predicting teleological acceptance. As belief in God increases, the effect of causality becomes stronger and more positive. Shaded areas represent $95 \%$ confidence intervals. 
Finally, we investigated whether a tendency to inhibit intuitions constrains teleological acceptance in the presence of a strong causal relationship. As shown in Table 6 (Model 4), probability of the conditional positively predicted acceptance of teleological explanations about biological and nonbiological natural entities, whereas inhibition of intuitions negatively predicted acceptance of teleological explanations about biological and nonbiological natural entities. As predicted, there was a significant interaction between probability of the conditional and inhibition of intuitions, showing that as inhibition of intuitions increased, the effect of causality on teleological acceptance decreased (see Figure 3). The likelihood of acceptance for teleological explanations about biological and nonbiological natural entities associated with a standard deviation increase in probability of the conditional (87\%), decreased by $11 \%$ for every standard deviation increase in inhibition of intuitions. Probability of the conditional also positively predicted acceptance of false teleological control statements. However, in contrast to explanations about biological and nonbiological natural entities, inhibition of intuitions did not negatively predict acceptance of false teleological control statements, and inhibition of intuitions did not constrain acceptance of false teleological control statements in the presence of a strong causal relationship. 
Figure 3. Teleological Acceptance as a Function of Causality and Inhibition of Intuitions

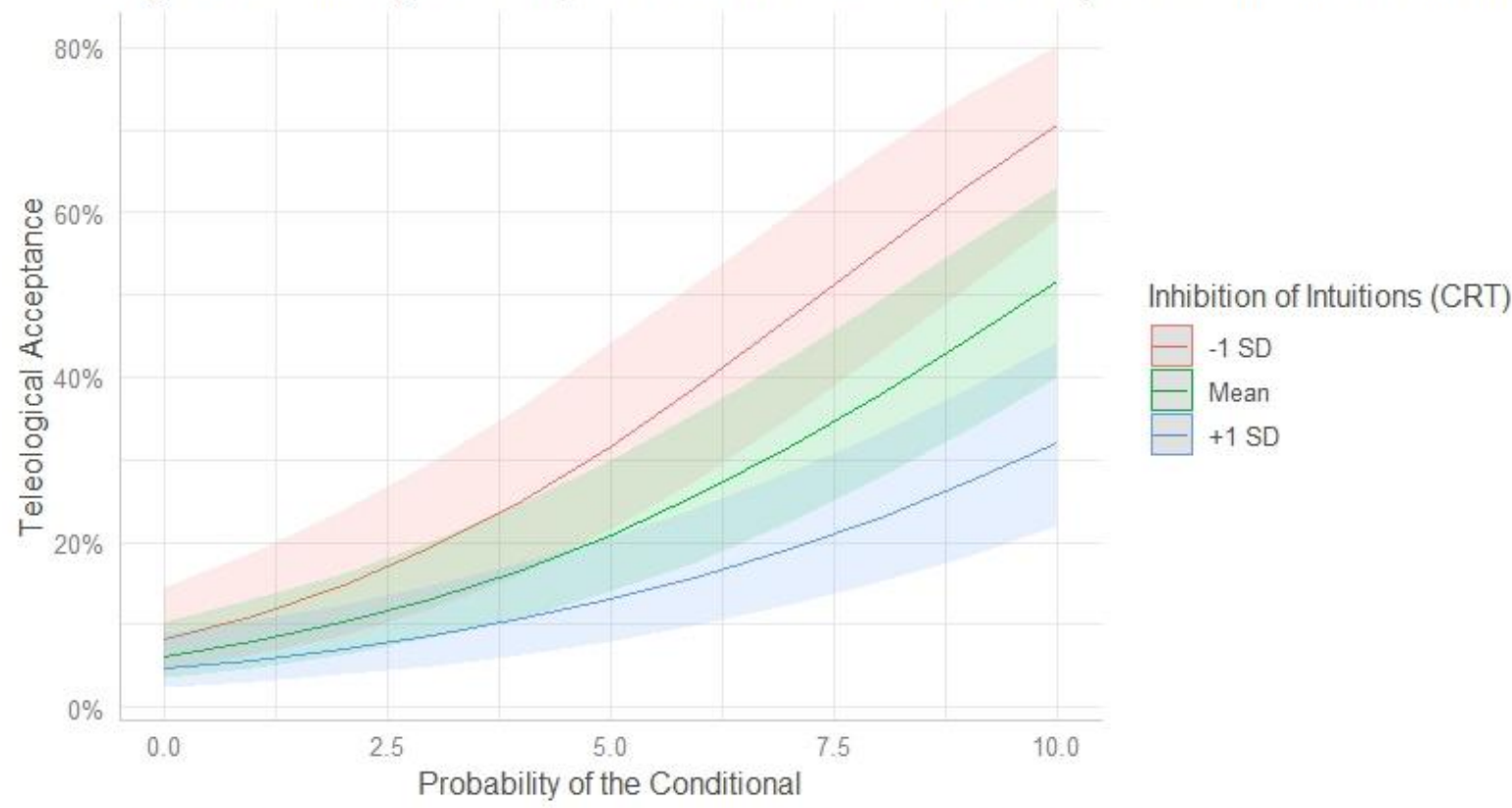

Note. Figure 3 shows the interaction between causality (probability of the conditional) and inhibition of intuitions (Cognitive Reflections Test) in predicting teleological acceptance. As inhibition of intuitions increases, the effect of causality becomes weaker and less positive. Shaded areas represent $95 \%$ confidence intervals.

\section{Discussion}

Bivariate correlations in Study Three revealed no significant relationships between causal beliefs and individual differences in anthropomorphism or belief in God. For content about biological and nonbiological natural entities and for content relating to explanations that were objectively false, probability of the conditional scores were not related to the tendency to inhibit intuitions, whereas delta-p scores were only weakly and negatively related to the tendency to inhibit intuitions. In other words, perceptions of causality were not associated with attributions of intentionality, and there was only weak evidence for a relationship between perceptions of causality and inhibition of intuitions. Consistent with the results of Study One, which looked at structure-function fit, there were no significant relationships between teleological acceptance and probability of the conditional or delta-p scores. However, explanations judged to be higher in causal strength were more readily accepted in their teleological form. This was the case both for teleological explanations about biological and nonbiological natural entities and for teleological explanations that are objectively false. These 
results provide strong support for the claim that causal beliefs are necessary but not sufficient for teleological acceptance.

Replicating previous findings, acceptance of teleological explanations about biological and nonbiological natural entities was positively related to anthropomorphism and belief in God (Roberts et al., 2021; Willard et al., 2020; Willard \& Norenzayan, 2013) and negatively related to the tendency to inhibit intuitions (Roberts et al., 2021; Zemla et al., 2012). Of primary interest, as attributions of intentionality increased, the effect of causal beliefs on acceptance of teleological explanations about biological and nonbiological natural entities became stronger and more positive, whereas as inhibition of intuitions increased, the effect of causal beliefs on acceptance of teleological explanations about biological and nonbiological natural entities became weaker and less positive. In contrast, individual differences in anthropomorphism, belief in God, and inhibition of intuitions, did not moderate the effect of causality on acceptance of teleological explanations that are objectively false. These findings suggest that the moderating effects of anthropomorphism, belief in God, and inhibition of intuitions on causal beliefs were not due to a general response bias. Rather, in combination, these findings provide compelling support for the hypothesis that attributions of intentionality facilitate the transition from a causal relationship to teleological beliefs about biological and nonbiological natural entities, and that inhibition of intuitions constrains this transition.

\section{General Discussion}

Everything happens for a reason, but reasons are sometimes domain specific. Although everything could, in principle, be explained with mechanistic reasons, certain things, such as intentional action or human-made artefacts, are better explained with teleological reasons (Dennett, 1987, 2017). However, research has shown that teleological reasons are sometimes applied to things which warrant only mechanistic reasons, such as biological and nonbiological natural entities (Casler \& Kelemen, 2007; Kelemen et al., 2013; Kelemen \& Rosset, 2009; 
Roberts et al., 2021). Across three studies, we tested the compatibility between two accounts of teleological reasoning: Kelemen's (1999a) intention-based account, and Liquin and Lombrozo's (2018) structure-function fit account. Study One showed support for both accounts, with judgements of structure-function fit and individual differences in anthropomorphism and inhibition of intuitions all significant predictors of teleological beliefs. Study Two explored what underlies judgements of structure-function fit and found, consistent with Wright (1976), that judgements of structure-function fit were strongly predicted by the perceived strength of causal relationship between a structure and a function. Finally, Study Three explored whether strong causal relationships were seen as more appropriate for teleological explanation, and whether individual differences in anthropomorphism and belief in God can facilitate the transition from a strong causal relationship to a teleological belief, and whether individual differences in the tendency to inhibit intuitions can constrain the transition from a strong causal relationship to a teleological belief. The results of Study Three supported this prediction, suggesting that Kelemen's intention-based account and Liquin and Lombrozo's structure-function fit account are compatible, and that both can be reconciled with Wright's analysis.

In support of the claim that teleological beliefs about biological and nonbiological natural entities may be facilitated by attributions of intentionality, and consistent with prior work (Kelemen et al., 2013; Roberts et al., 2021; Willard et al., 2020; Willard \& Norenzayan, 2013), in Study One, we found some evidence to suggest that highly anthropomorphic individuals were more accepting of such teleological explanations. Study Three provided stronger evidence in support of this relationship, with anthropomorphism a highly significant and positive predictor of teleological acceptance. Also consistent with prior work (Järnefelt et al., 2015; Kelemen, 2004; Kelemen \& Diyanni, 2005), Study Three showed that individuals who expressed greater belief in God were more accepting of teleological explanations. As 
teleological explanations about biological and nonbiological natural entities lack an agent to which intentions can be attributed, overall, these results suggest that acceptance of such teleological explanations reflect a belief that the natural world is either designed by an intending creator or is itself capable of intentional action.

Consistent with Kelemen et al.'s (2013, p. 1075) claim that teleological reasoning is a 'developmentally persistent cognitive default', much of the research in this field has situated the expression of teleological beliefs within a dual-process framework (e.g., Banerjee \& Bloom, 2014; Kelemen \& Rosset, 2009; Lombrozo et al., 2007). According to this perspective, teleological beliefs about biological and nonbiological natural entities are the result of an automatic cognitive process, and to inhibit this automatic cognitive process requires the engagement of effortful thought (e.g., De Neys, 2014; Evans, 2010; Pennycook et al., 2015). Using the Cognitive Reflection Test as a measure of the tendency to inhibit intuitions, in Studies One and Three, we found that individuals who tended to inhibit intuitively appealing incorrect responses to simple reasoning problems, were less accepting of teleological explanations about biological and nonbiological natural entities. This replicates prior findings (Roberts et al., 2021; Zemla et al., 2012), and supports the view that certain teleological explanations are intuitively appealing.

Although some teleological explanations about biological and nonbiological natural entities may be intuitively appealing, explanations vary in how appealing they are. Replicating Liquin and Lombrozo (2018, study 5), in Study One, structure-function fit was a strong, positive, and significant predictor of teleological acceptance. However, bivariate correlations provided no evidence to suggest that individuals who rated structure-function fit as being higher were more accepting of such teleological explanations on average. Using two measures of causal belief - probability of the conditional and delta-p - Study Two found that a belief that a function would occur in the presence of a structure (i.e., a biological or nonbiological 
natural entity) was strongly related to judgements of structure-function fit, suggesting that questions about structure-function fit are interpreted as asking whether a structure plays a causal role in bringing about a function. Study Three showed that teleological explanations in which there was a strong causal relationship between an entity and a function were most likely to be accepted as true, but again, individuals who rated causal relationships as stronger were no more accepting of teleological explanations on average. Certain teleological explanations about biological and nonbiological natural entities are seen as more acceptable than others, but these findings suggest that a strong causal relationship between an entity and a function may be necessary but not sufficient for teleological acceptance to occur.

Causality appears to be necessary but not sufficient for teleology, but the key findings from the present studies show how Liquin and Lombrozo's (2018) structure-function fit account is compatible with Kelemen's (1999a) intention-based account, and how both can be reconciled with Wright's (1976) analysis. Teleological explanations of intentional design are appropriate not only because a function is a consequence of an artefact being there, but also because the intentions of a designer mean an artefact could be said to exist because it results in a certain function. Similarly, teleological explanations of intentional action are appropriate not only because a behaviour tends to bring about a goal, but also because the intentions of an agent mean that a behaviour could be said to occur because it tends to bring about a goal. In both cases, the intentions of an agent mean Wright's (1976) second criterion can be met. In the context of teleological explanations about biological and nonbiological natural entities, objectively, the second criterion appears not to be met, as no agent intends for the specified function or goal to occur. In Study One, we found that the previously reported positive relationship between teleological acceptance and anthropomorphism (Kelemen et al., 2013; Roberts et al., 2021; Willard et al., 2020; Willard \& Norenzayan, 2013), was strongest for statements judged to have high structure-function fit. Using two measures of causal beliefs - 
probability of the conditional and delta-p - Study Three replicated the findings from Study One, with the effect of causal strength on teleological acceptance becoming stronger and more positive as anthropomorphic tendencies increased. Study Three found a similar pattern of results with the previously reported positive relationship between teleological acceptance and belief in God (Järnefelt et al., 2015; Kelemen, 2004; Kelemen \& Diyanni, 2005). Again, the effect of causal strength on teleological acceptance became stronger and more positive as belief in God increased. These findings suggest that a willingness to attribute intentions, either to a biological or nonbiological natural entity itself, or to an agent believed to be the designer of the entity, allow Wright's (1976) second criterion can be met. As such, attributions of intentionality may facilitate the transition from a strong causal relationship to teleological acceptance.

\section{Strengths and Limitations}

A possible objection to the claim that causality is necessary but not sufficient for teleology, is that causal beliefs were not measured directly in the present studies. That is, perhaps ratings of probability of the conditional and delta-p were measuring correlational beliefs rather than causal beliefs. Although the current results cannot rule out this possibility, prior research shows that the relationship between probability of the conditional and explicit judgements of causality is very strong (Over et al., 2007). The correlational account also seems unlikely given Wright's (1976) formulation. Wright (1976) argues that for teleological explanations about goal directed behaviour, the behaviour needs to bring about the goal, whereas for human-made artefacts, the function needs to be a consequence of the artefact being there. Consider the teleological explanation "doctors prescribe antibiotics in order to treat infections". For this explanation to be true, it requires, at minimum, that doctors believe the prescription of antibiotics will play a causal role in treating infection. Otherwise, we could only conclude that "doctors prescribe antibiotics and infections are treated". 
A second possible objection to the claim that causality is necessary but not sufficient for teleology, is that perhaps it is teleology that is necessary but not sufficient for causality. That is, because causality was not experimentally manipulated in the current studies, it is difficult to make claims about the direction of this relationship. However, the results from Studies Two and Three support the claim that causality is necessary for teleology and not the other way around. Both studies showed that people can hold causal beliefs in false control statements, even though these statements are accepted at close to floor levels in their teleological form (Kelemen et al., 2013; Roberts et al., 2021). If teleology was necessary for causality, people should not hold causal beliefs in these statements.

\section{Implications and Future Directions}

In showing the compatibility between psychological (Kelemen, 1999a; Liquin \& Lombrozo, 2018) and philosophical accounts (Wright, 1976) of teleological reasoning, the present findings are of both theoretical and practical significance. The theoretical significance of these findings is that they support the view that acceptance of teleological explanations about biological and nonbiological natural entities should be considered causal from a descriptive perspective (Kelemen, 1999a, 2004). The increased rate of teleological acceptance amongst highly religious or highly anthropomorphic individuals was most pronounced in cases where Wright's first criterion was clearly met. In the presence of a strong causal relationship, if an individual believes a biological or nonbiological natural entity is capable of goal-directed behaviour or was designed by an intending creator to fulfil a specific function, then for that individual, acceptance of teleological explanations in these domains appears to be based on the same considerations as acceptance of teleological explanations that are considered normatively correct. Therefore, although acceptance of teleological explanations about biological and nonbiological natural entities may be a deviation from what is considered normative, based on the individual's beliefs, it should not necessarily be considered an error in reasoning. 
Teleological beliefs about biological and nonbiological natural entities are sometimes considered diametrically opposed to a scientific, mechanistic worldview (Talanquer, 2007, 2013; Wicken, 1981). As such, there may be an argument in favour of reducing rates of teleological acceptance within the biological and nonbiological natural domain in favour of promoting scientific understanding. The practical significance of these findings is that they suggest there may be three distinct pathways to modify existing teleological beliefs within this domain. First, it may be possible to modify teleological beliefs by challenging religious or anthropomorphic beliefs. In support of this, cross-sectional studies show that acceptance of teleological explanations about biological and nonbiological natural entities are positively related to religious and anthropomorphic beliefs (Järnefelt et al., 2015; Kelemen et al., 2013; Kelemen \& Diyanni, 2005; Liquin \& Lombrozo, 2018; Roberts et al., 2020, 2021; Willard et al., 2020; Willard \& Norenzayan, 2013). Furthermore, experimental evidence shows that rates of teleological acceptance are lower when people are told that a structure came about because of non-intentional compared to intentional human design (Lombrozo \& Carey, 2006). Applying this to teleological explanations about biological and nonbiological natural entities which are facilitated by religious and anthropomorphic beliefs, in principle, it could be possible to reduce teleological acceptance by challenging a person's belief that an agent (whether an intending creator or the entity itself) acted intentionally. However, given religious and anthropomorphic (i.e., Mother Nature or Gaia) beliefs are argued to result from ordinary cognitive processes operating in normal human environments (Atran, 2002; Barrett, 2000; Barrett \& Lanman, 2008; Boyer, 1994), and given that the tendency to adopt a design stance or intentional stance arises so early in development (Casler \& Kelemen, 2005; Csibra, 2008; Gergely et al., 1995; Hernik \& Csibra, 2015; Johnson et al., 2001; Meltzoff, 1995), these beliefs may be resistant to change. Furthermore, as such beliefs are often central to an individual's 
identity, attempting to modify teleological beliefs by challenging such deeply held worldviews may not only prove ineffective, but ethically dubious.

Second, it may be possible to modify teleological beliefs by challenging an individual's preconceived belief in the strength of a causal relationship. The present studies suggest that this would be possible in principle. However, paradoxically, this approach seems to be the antithesis of what modifying teleological beliefs would aim to achieve. As scientific explanations of the world preference antecedent causal physical processes, challenging an individual's belief in a causal process may indeed result in decreased teleological acceptance, but it is also likely to impede scientific understanding of the natural world. Conversely, although teleological acceptance has been shown to decrease with formal education (Casler \& Kelemen, 2008; Sánchez Tapia et al., 2016), evidence suggests that this is likely due to the inhibition, rather than extinction of teleological tendencies (Kelemen et al., 2013; Kelemen \& Rosset, 2009; Lombrozo et al., 2007; Mills \& Frowley, 2014; Roberts et al., 2020). Therefore, although scientific knowledge may temporarily reduce teleological acceptance, ironically, by emphasising the causal connections between things in the world, this may also make them better candidates for teleological explanation.

Finally, a novel approach to modifying teleological beliefs could be to focus not on challenging an individual's beliefs about intentionality or causality in the natural world, but instead, in combination with scientific education, to teach a unified philosophical and psychological account of teleological reasoning itself. This would involve informing people that strong causal relationships make good candidates for teleological explanation, and that religious and anthropomorphic beliefs may facilitate acceptance of such explanations. Furthermore, by teaching people about the philosophical commitments underlying teleological explanation, people may be more inclined to reflect upon their intuitions before accepting such explanations. This approach would avoid the practical and ethical drawbacks associated with 
attempting to reduce teleological beliefs by focusing directly on either of Wright's (1976) criteria. By making explicit the assumptions underlying acceptance of teleological explanations about biological and nonbiological natural entities, individuals may be better able to determine whether they agree with such assumptions.

\section{Conclusion}

The present studies have demonstrated that Liquin and Lombrozo's (2018) structurefunction fit account of teleological reasoning is compatible with Kelemen's (1999a) intentionbased account, and that both can be reconciled with Wright's (1976) analysis. Our results show that a causal link between a structure and a function is a necessary but not sufficient condition for a teleology, and that attributions of intentionality can facilitate the transition from a causal link to a teleological belief. The relationships between teleological acceptance and both religious (Järnefelt et al., 2015; Kelemen, 2004) and anthropomorphic beliefs (Kelemen et al., 2013; Roberts et al., 2021; Willard et al., 2020; Willard \& Norenzayan, 2013) appear to be more nuanced than previously thought. These findings show the evaluation of teleological explanations about biological and nonbiological natural entities is based on the same underlying considerations as teleological explanations about intentional action and intentional design, and as such, demonstrate the psychological reality of Wright's (1976) analysis in this context. 


\section{References}

Atran, S. (2002). In Gods we trust. Oxford University Press.

Atran, S., \& Norenzayan, A. (2004). Religion's evolutionary landscape: Counterintuition, commitment, compassion, communion. Behavioral and Brain Sciences, 27(2004), 1-67.

Ayala, F. J. (2007). Darwin's greatest discovery: Design without designer. In the Light of Evolution, 1, 3-21. https://doi.org/10.17226/11790

Banerjee, K., \& Bloom, P. (2014). Why did this happen to me? Religious believers' and nonbelievers' teleological reasoning about life events. Cognition, 133(1), 277-303. https://doi.org/10.1016/j.cognition.2014.06.017

Barnes, M. E., Evans, E. M., Hazel, A., Brownell, S. E., \& Nesse, R. M. (2017). Teleological reasoning, not acceptance of evolution, impacts students' ability to learn natural selection. Evolution: Education and Outreach, 10(1). https://doi.org/10.1186/s12052017-0070-6

Barrett, J. L. (2000). Exploring the natural foundations of religion. Trends in Cognitive Sciences, 4(1), 29-34. https://doi.org/10.1016/S1364-6613(99)01419-9

Barrett, J. L., \& Lanman, J. A. (2008). The science of religious beliefs. Religion, 38(2), 109124. https://doi.org/10.1016/j.religion.2008.01.007

Bates, D., Mächler, M., Bolker, B. M., \& Walker, S. C. (2015). Fitting linear mixed-effects models using lme4. Journal of Statistical Software, 67(1). https://doi.org/10.18637/jss.v067.i01

Boyer, P. (1994). The naturalness of religious ideas: A cognitive theory of religion. University of California Press.

Brumby, M. N. (1984). Misconceptions about the concept of natural selection by medical biology students. Science Education, 68(4), 493-503. https://doi.org/10.1002/sce.3730680412 
Casler, K., \& Kelemen, D. (2005). Young children's rapid learning about artifacts. Developmental Science, 6, 472-480. https://doi.org/10.1111/j.1467-7687.2005.00438.x

Casler, K., \& Kelemen, D. (2007). Reasoning about artifacts at 24 months: The developing teleo-functional stance. Cognition, 103(1), 120-130.

https://doi.org/10.1016/j.cognition.2006.02.006

Casler, K., \& Kelemen, D. (2008). Developmental continuity in teleo-functional explanation: Reasoning about nature among Romanian Romani adults. Journal of Cognition and Development, 9(3), 340-362. https://doi.org/10.1080/15248370802248556

Cheng, P. W. (1997). From covariation to causation: A causal power theory. Psychological Association, Inc, 104(2), 367-405.

Csibra, G. (2008). Goal attribution to inanimate agents by 6.5 -month-old infants. Cognition, 107(2), 705-717. https://doi.org/10.1016/j.cognition.2007.08.001

De Neys, W. (2014). Dual process theory 2.0 (W. De Neys (ed.)). Routledge.

Dennett, D. C. (1987). The intentional stance. MIT Press.

Dennett, D. C. (2017). From bacteria to Bach and back. W. W. Norton \& Company.

Evans, J. S. B. T. (2010). Intuition and Reasoning: A Dual-Process Perspective. Psychological Inquiry, 21(4), 313-326. https://doi.org/10.1080/1047840X.2010.521057

Evans, J. S. B. T., Handley, S. J., Hadjchristidis, C., Thompson, V., Over, D. E., \& Bennett, S. (2007). On the basis of belief in causal and diagnostic conditionals. Quarterly Journal of Experimental Psychology, 60(5), 635-643. https://doi.org/10.1080/17470210601100274

Frederick, S. (2005). Cognitive reflection and decision making. Journal of Economic Perspectives, 19(4), 25-42. https://doi.org/10.1257/089533005775196732

Gergely, G., Nádasdy, Z., Csibra, G., \& Bíró, S. (1995). Taking the intentional stance at 12 months of age. Cognition, 56(2), 165-193. https://doi.org/10.1016/0010- 
0277(95)00661-H

Green, P., \& Macleod, C. J. (2016). SIMR: An R package for power analysis of generalized linear mixed models by simulation. Methods in Ecology and Evolution, 7(4), 493-498. https://doi.org/10.1111/2041-210X.12504

Greif, M. L., Kemler Nelson, D. G., Keil, F. C., \& Gutierrez, F. (2006). What do children want to know about animals and artifacts? Domain-specific requests for information. Psychological Science, 17(6), 455-459. https://doi.org/10.1111/j.14679280.2006.01727.x

Hempel, C. G., \& Oppenheim, P. (1948). Studies in the logic of explanation. Philosophy of Science, 15(2), 135-175.

Hernik, M., \& Csibra, G. (2015). Infants learn enduring functions of novel tools from action demonstrations. Journal of Experimental Child Psychology, 130, 176-192. https://doi.org/10.1016/j.jecp.2014.10.004

Huber, S., \& Huber, O. W. (2012). The centrality of religiosity scale (CRS). Religions, 3, 710-724. https://doi.org/10.3390/rel3030710

Järnefelt, E., Canfield, C. F., \& Kelemen, D. (2015). The divided mind of a disbeliever: Intuitive beliefs about nature as purposefully created among different groups of nonreligious adults. Cognition, 140, 72-88. https://doi.org/10.1016/j.cognition.2015.02.005 Johnson, S. C., Booth, A., \& O’Hearn, K. (2001). Inferring the goals of a nonhuman agent. Cognitive Development, 16(1), 637-656. https://doi.org/10.1016/S0885-2014(01)000430

Keil, F. C. (1992). The origins of an autonomous biology. In M. R. Gunnar \& M. Maratsos (Eds.), Modularity and constraints in language and cognition (pp. 103-137). Lawrence Erlbaum Associates.

Keil, F. C. (1995). The growth of causal understandings of natural kinds. In D. Sperber, D. 
Premack, \& A. J. Premack (Eds.), Causal cognition: A multidisciplinary debate (pp. 234-267). Clarendon Press.

Kelemen, D. (1999a). Beliefs about purpose: On the origins of teleological thought. In M. C. Corballis \& S. E. G. Lea (Eds.), The descent of mind: Psychological perspectives on hominid evolution (pp. 278-310). Oxford University Press.

Kelemen, D. (1999b). Function, goals and intention: Children's teleological reasoning about objects. Trends in Cognitive Sciences, 3(12), 461-468. https://doi.org/10.1016/S13646613(99)01402-3

Kelemen, D. (1999c). The scope of teleological thinking in preschool children. Cognition, 70(3), 241-272. https://doi.org/10.1016/S0010-0277(99)00010-4

Kelemen, D. (1999d). Why are rocks pointy? Children's preference for teleological explanations of the natural world. Developmental Psychology, 35(6), 1440-1452. https://doi.org/10.1037/0012-1649.35.6.1440

Kelemen, D. (2004). Are children "intuitive theists"? Reasoning about purpose and design in nature. Psychological Science, 15(5), 295-301. https://doi.org/10.1111/j.09567976.2004.00672.x

Kelemen, D., \& Diyanni, C. (2005). Intuitions about origins: Purpose and intelligent design in children's reasoning about nature. Journal of Cognition and Development, 6(1), 3-31. https://doi.org/10.1207/s15327647jcd0601

Kelemen, D., \& Rosset, E. (2009). The Human function compunction: Teleological explanation in adults. Cognition, 111(1), 138-143. https://doi.org/10.1016/j.cognition.2009.01.001

Kelemen, D., Rottman, J., \& Seston, R. (2013). Professional physical scientists display tenacious teleological tendencies: Purpose-based reasoning as a cognitive default. Journal of Experimental Psychology: General, 142(4), 1074-1083. 
https://doi.org/10.1037/a0030399

Liquin, E. G., \& Lombrozo, T. (2018). Structure-function fit underlies the evaluation of teleological explanations. Cognitive Psychology, 107(October), 22-43. https://doi.org/10.1016/j.cogpsych.2018.09.001

Lombrozo, T., Bonawitz, E. B., \& Scalise, N. R. (2018). Young children's learning and generalization of teleological and mechanistic explanations. Journal of Cognition and Development, 19(2), 220-232. https://doi.org/10.1080/15248372.2018.1427099

Lombrozo, T., \& Carey, S. (2006). Functional explanation and the function of explanation. Cognition, 99(2), 167-204. https://doi.org/10.1016/j.cognition.2004.12.009

Lombrozo, T., Kelemen, D., \& Zaitchik, D. (2007). Inferring design: Evidence for a preference for teleological explanations in patients with Alzheimer's disease. Psychological Science, 18(11), 999-1007.

Meltzoff, A. N. (1995). Understanding the intentions of others: Re-enactment of intentions by 18-months-old children. Developmental Psychology, 31(September), 838-850. https://doi.org/10.1037/0012-1649.31.5.838

Mills, R., \& Frowley, J. (2014). Promiscuous Teleology and the effect of Locus of Control. Irish Journal of Psychology, 35(2-3), 121-132. https://doi.org/10.1080/03033910.2015.1011192

Ojalehto, B., Waxman, S. R., \& Medin, D. L. (2013). Teleological reasoning about nature: Intentional design or relational perspectives? Trends in Cognitive Sciences, 17(4), 166171. https://doi.org/10.1016/j.tics.2013.02.006

Over, D. E. (2017). Causation and the probability of causal conditionals. In The Oxford Handbook of Causal Reasoning (Vol. 1, Issue October 2018, pp. 1-34). https://doi.org/10.1093/oxfordhb/9780199399550.013.3

Over, D. E., Hadjichristidis, C., Evans, J. S. B. T., Handley, S. J., \& Sloman, S. A. (2007). 
The probability of causal conditionals. Cognitive Psychology, 54(1), 62-97. https://doi.org/10.1016/j.cogpsych.2006.05.002

Pennycook, G., Fugelsang, J. A., \& Koehler, D. J. (2015). What makes us think? A threestage dual-process model of analytic engagement. Cognitive Psychology, 80, 34-72. https://doi.org/10.1016/j.cogpsych.2015.05.001

Pennycook, G., \& Rand, D. G. (2019). Cognitive Reflection and the 2016 U.S. Presidential Election. Personality and Social Psychology Bulletin, 45(2), 224-239. https://doi.org/10.1177/0146167218783192

Roberts, A. J., Handley, S. J., \& Polito, V. (2021). The design stance, intentional stance, and teleological beliefs about biological and non-biological natural entites. Journal of Personality and Social Psychology, 120(6), 1720-1748. https://doi.org/10.1037/pspp0000383

Roberts, A. J., Wastell, C. A., \& Polito, V. (2020). Teleology and the intentions of supernatural agents. Consciousness and Cognition, 80(February), 102905. https://doi.org/10.1016/j.concog.2020.102905

Sánchez Tapia, I., Gelman, S. A., Hollander, M. A., Manczak, E. M., Mannheim, B., \& Escalante, C. (2016). Development of Teleological Explanations in Peruvian QuechuaSpeaking and U.S. English-Speaking Preschoolers and Adults. Child Development, 87(3), 747-758. https://doi.org/10.1111/cdev.12497

Talanquer, V. (2007). Explanations and teleology in chemistry education. International Journal of Science Education, 29(7), 853-870. https://doi.org/10.1080/09500690601087632

Talanquer, V. (2013). When atoms want. Journal of Chemical Education, 90(11), 14191424. https://doi.org/10.1021/ed400311x

Thomson, K. S., \& Oppenheimer, D. M. (2016). Investigating an alternate form of the 
cognitive reflection test. Judgment and Decision Making, 11(1), 99-113. https://doi.org/ISBN 978-615-5270-17-8

Waytz, A., Cacioppo, J. T., \& Epley, N. (2010). Who sees human? The stability and importance of individual differences in anthropomorphism. Perspectives on Psychological Science, 5(3), 219-232. https://doi.org/10.1177/1745691610369336

Wicken, J. S. (1981). Causal explanations in classical and statistical thermodynamics. Philosophy of Science, 48(1), 65-77.

Willard, A. K., Cingl, L., \& Norenzayan, A. (2020). Cognitive Biases and Religious Belief: A Path Model Replication in the Czech Republic and Slovakia With a Focus on Anthropomorphism. Social Psychological and Personality Science, 11(1), 97-106. https://doi.org/10.1177/1948550619841629

Willard, A. K., \& Norenzayan, A. (2013). Cognitive biases explain religious belief, paranormal belief, and belief in life's purpose. Cognition, 129(2), 379-391. https://doi.org/10.1016/j.cognition.2013.07.016

Wright, L. (1976). Teleological explanations. University of California Press.

Zemla, J. C., Steiner, S. M., \& Sloman, S. (2012). Analytical Thinking Predicts Less Teleological Reasoning and Religious Belief. Proceedings of the 38th Annual Meeting of the Cognitive Science Society, August, 1217-1222. 